$1-6-2000$

\title{
Structural Damage Detection Using Modal Norms
}

\author{
W. Gawronski \\ California Institute of Technology \\ Jerzy T. Sawicki \\ Cleveland State University, j.sawicki@csuohio.edu
}

Follow this and additional works at: https://engagedscholarship.csuohio.edu/enme_facpub

Part of the Mechanical Engineering Commons

How does access to this work benefit you? Let us know!

\section{Publisher's Statement}

NOTICE: this is the author's version of a work that was accepted for publication in Journal of Sound and Vibration. Changes resulting from the publishing process, such as peer review, editing, corrections, structural formatting, and other quality control mechanisms may not be reflected in this document. Changes may have been made to this work since it was submitted for publication. A definitive version was subsequently published in Journal of Sound and Vibration, 229, 1, (01-06-2000); 10.1006/jsvi.1999.2179

\section{Original Citation}

Gawronski, W., and Sawicki, J.T. (2000) Structural Damage Detection Using Modal Norms. Journal of Sound and Vibration, 229(1), 194-198, doi: 10.1006/jsvi.1999.2179.

This Article is brought to you for free and open access by the Mechanical Engineering Department at EngagedScholarship@CSU. It has been accepted for inclusion in Mechanical Engineering Faculty Publications by an authorized administrator of EngagedScholarship@CSU. For more information, please contact library.es@csuohio.edu. 


\title{
STRUCTURAL DAMAGE DETECTION USING MODAL NORMS
}

\author{
W. GAWRONSKI \\ Jet Propulsion Laboratory, California Institute of Technology, Pasadena, CA 91109, U.S.A. \\ AND \\ J. T. SAWICKI \\ Cleveland State University, Department of Mechanical Engineering, Cleveland, OH 44115, \\ U.S.A.
}

\section{INTRODUCTION}

Different approaches and criteria are used to sense and to localize a damage of flexible structures. Most of the methods take advantage of the dynamics behavior of the structural model [1-6]. This paper uses the modal and sensor norms, as defined in reference [7], to determine damage locations. The proposed approach allows localization of damaged elements of a structure, and provides the information about the impact of the damage on the natural modes of the damaged structure.

\section{MODAL MODEL}

A structural model is characterized by its mass, stiffness, and damping matrices, as well as by the locations of the sensors and actuators. It is represented by the following second

$$
M \ddot{q}+D \dot{q}+K q=B u, \quad y=C_{q} q+C_{v} \dot{q} .
$$

In this equation $q$ is the structural displacement vector of dimension $n_{d}, u$ is the input vector of dimension $r, y$ is the output vector of dimension $s$, and $M, D, K$ are the mass, damping, and stiffness matrices, respectively, of dimensions $n_{d} \times n_{d}$. The input matrix $B$ of dimensions $n_{d} \times r$, characterizes the actuator locations, the output displacement and rate matrices $C_{q}$ and $C_{v}$ of dimensions $s \times n_{d}$ characterize the displacement and rate sensor locations. The mass matrix is positive definite, and the stiffness and damping matrices are positive semidefinite; $n_{d}$ is the number of degrees of freedom, $r$ is the number of actuators, and $s$ is the number of sensors.

The above equation is also obtained in the modal co-ordinates using the modal transformation. For a small proportional damping let $\omega_{i}$ be the $i$ th natural frequency and $\phi_{i}$ be the $i$ th natural mode, or mode shape. Define the matrix of natural 
frequencies $\Omega=\operatorname{diag}\left(\omega_{1}, \omega_{2}, \ldots, \omega_{n}\right)$. and the modal matrix $\Phi=\left[\phi_{1} \phi_{2} \ldots \phi_{n}\right]$, of dimensions $n_{d} \times n$, consists of $n$ natural modes of a structure. A new variable, $q_{m}$, called the modal displacement vector is introduced, such that $q=\Phi q_{m}$. This transformation produces the modal mass matrix $M_{m}=\Phi^{T} M \Phi$, and the modal stiffness matrix $K_{m}=\Phi^{T} K \Phi$. Both matrices are diagonal. If the damping is proportional, the modal damping matrix $D_{m}=\Phi^{T} D \Phi$ is diagonal as well.

Left-multiplying equation (1) by $\Phi^{T}$, and subsequently by $M_{m}^{-1}$ gives the modal model

$$
\ddot{q}_{m}+2 Z \Omega \dot{q}_{m}+\Omega^{2} q_{m}=B_{m} u, \quad y=C_{m q} q_{m}+C_{m v} \dot{q}_{m},
$$

where $Z=0.5 M_{m}^{-1} D_{m} \Omega^{-1}$ is a diagonal matrix of the modal damping, and $B_{m}$ is the modal input matrix, $B_{m}=M_{m}^{-1} \Phi^{T} B$, while $C_{m q}=C_{q} \Phi$ and $C_{m v}=C_{v} \Phi$ are the modal displacement and rate matrices, respectively.

The modal equations ( $2 \mathrm{a}, \mathrm{b}$ ) can be re-written as a set of $n$ independent equations for each modal displacement

$$
\begin{gathered}
\ddot{q}_{m i}+2 \zeta_{i} \omega_{i} \dot{q}_{m i}+\omega_{i}^{2} q_{m i}=b_{m i} u, \\
y_{i}=c_{m q i} q_{m i}+c_{m v i} \dot{q}_{m i}, \quad i=1, \ldots, n,
\end{gathered}
$$

where $\zeta_{i}$ is the $i$ th diagonal entry of $Z$. In the above equations $y_{i}$ is the system output due to the $i$ th mode dynamics, while $b_{m i}$ is the $i$ th row of $B_{m}$, and $c_{m q i}$ and $c_{m v i}$ are the $i$ th columns of $C_{m q}$, and $C_{m v}$, respectively. Define $c_{m i}$ as the equivalent output matrix of the $i$ th mode,

$$
c_{m i}=c_{m q i} / \omega_{i}+c_{m v i}
$$

then $\left\|b_{m i}\right\|_{2}$ and $\left\|c_{m i}\right\|_{2}$ are the input and output gains of the $i$ th mode, see reference [7], where $\|x\|_{2}$ denotes the Frobenius norm of $x$, i.e., $\|x\|_{2}=\sqrt{\operatorname{tr}\left(x^{T} x\right)}$. In the case of multiple sensors $c_{m i}$ consists of $s$ entries $c_{m i j}, j=1, \ldots, s$, that correspond to each sensor.

\section{MODAL NORMS}

The $\mathrm{H}_{2}$ norm of a system is used to characterize the system itself, along with its modes, and its sensors. Let $G(\omega)$ be a transfer function of a system. The $\mathrm{H}_{2}$ norm of the system is defined as

$$
\|G\|_{2}^{2}=\frac{1}{2 \pi} \int_{-\infty}^{+\infty} \operatorname{tr}\left(G^{*}(\omega) G(\omega)\right) \mathrm{d} \omega
$$

In modal co-ordinates the equations of a flexible structure are uncoupled, see reference (3). In this case the norms of the ith mode and $j$ th sensor can be approximately determined from the following equations, cf. reference [7]:

$$
\left\|G_{i j}\right\|_{2} \cong\left\|b_{m i}\right\|_{2}\left\|c_{m i j}\right\|_{2} / 2 \sqrt{\zeta_{i} \omega_{i}}
$$




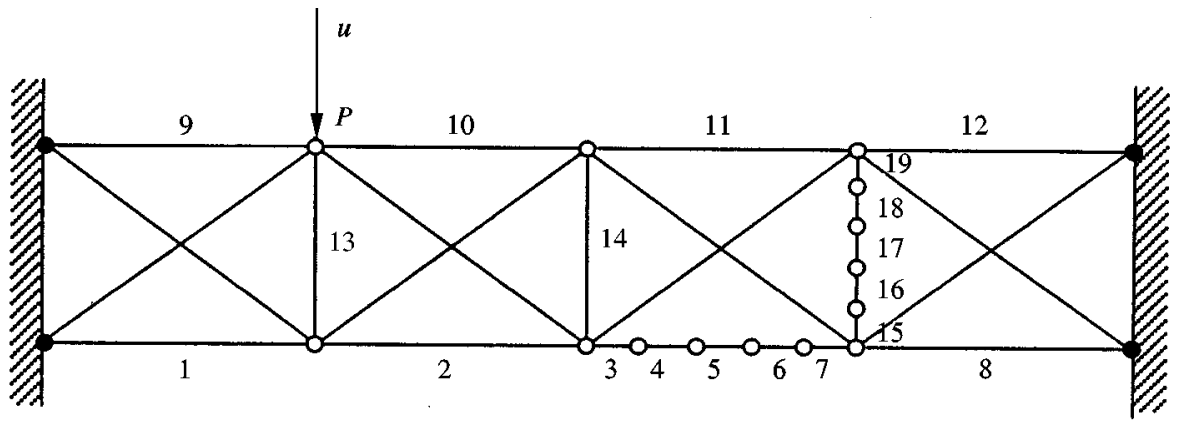

Figure 1. The beam structure: fixed nodes are filled, numbers refer to the sensors.

Define the $\mathrm{H}_{2}$ norm of the $j$ th mode, $G_{m i}$, as the root-mean-square sum over all sensors [7]:

$$
G_{m i} \stackrel{2}{2}=\sum_{=1}^{S} G_{i j} \stackrel{2}{2}, \quad i=1, \ldots, n .
$$

Define also the $\mathrm{H}_{2}$ norm of the $j$ th sensor, $G_{s}{ }_{2}$, as the root-mean-square sum over all modes,

$$
G_{s} \quad \stackrel{2}{2}=\sum_{i=1}^{n} G_{i} \quad \stackrel{2}{2}, \quad j=1, \ldots, s
$$
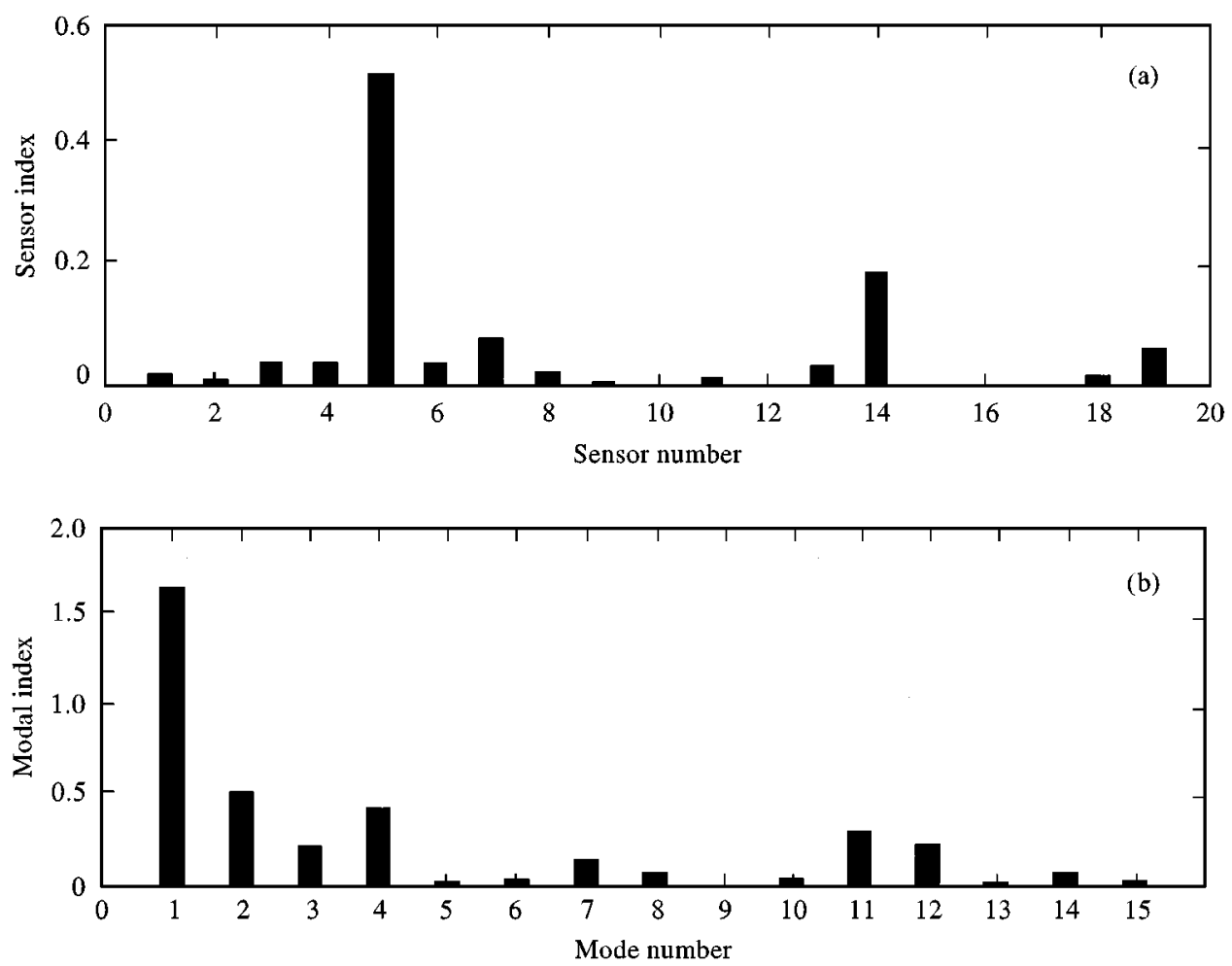

Figure 2. Sensor and modal indices for the beam structure, damage case 1. 

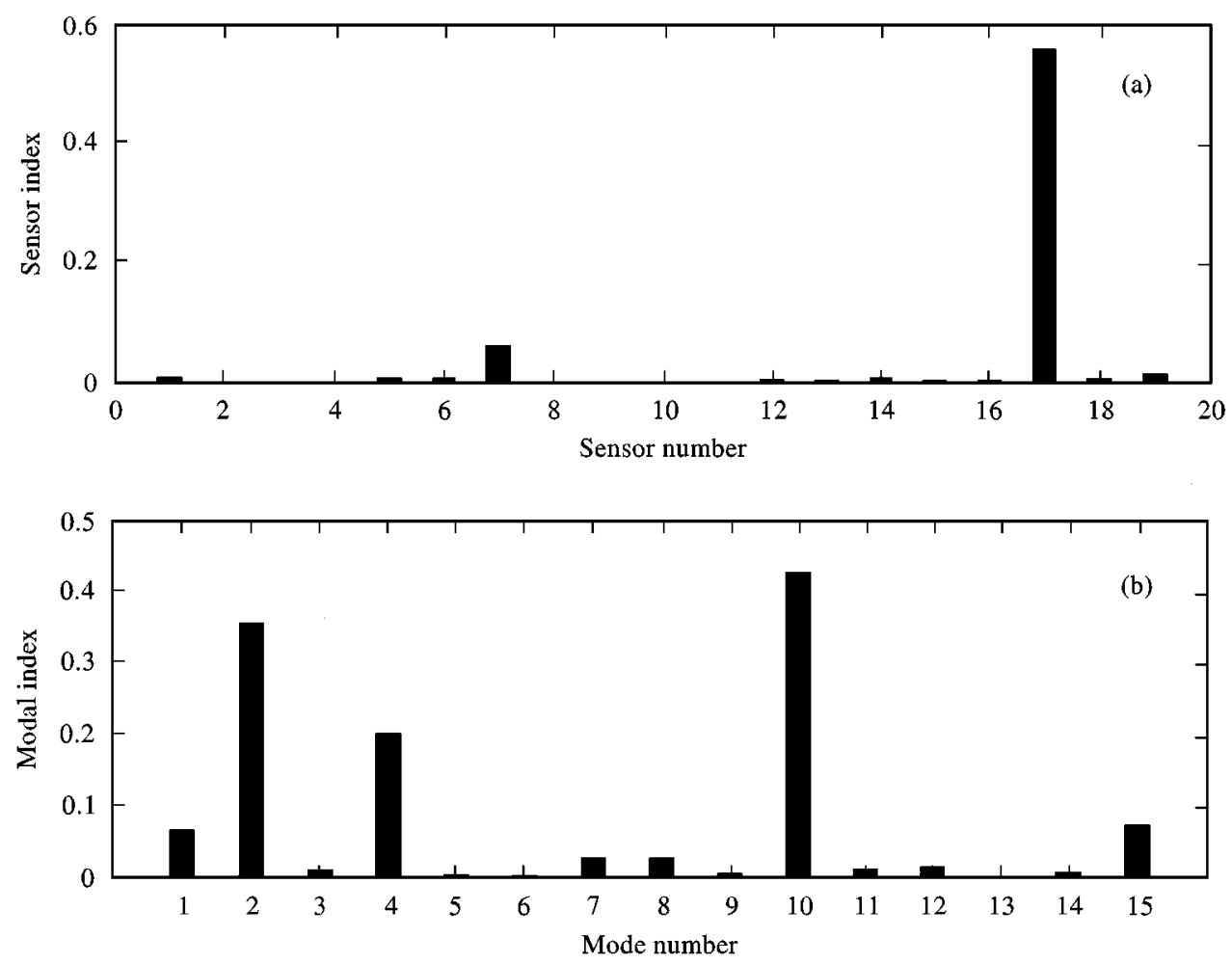

Figure 3. Sensor and modal indices for the beam structure, damage case 2.

The $\mathrm{H}_{2}$ norm of the total system is approximately the root-mean-square sum over either all its modal norms, or all its sensor norms, that is

$$
G_{2}^{2} \cong \sum_{i=1}^{n} G_{m i} 2 \text { or } \quad G_{2}^{2} \cong \sum_{=1}^{s} G_{s j} \frac{2}{2}
$$

The modal and sensor norms are used in the following damage detection procedure.

\section{DAMAGE DETECTORS}

Denote the $j$ th sensor norm of a healthy structure by $G_{s h} \quad 2$, and the $j$ th sensor norm of a damaged structure by $G_{s d j} 2$. The $j$ th sensor index of the structural damage is defined as a weighted difference between the $j$ th sensor norm of a healthy and damaged structure, i.e.,

$$
\sigma_{s j}=\left|\| G_{s h j} \frac{2}{2}-G_{s d j} \frac{2}{2}\right| / G_{s h} \frac{2}{2} .
$$

The sensor index reflects the impact of the structural damage on the $j$ th sensor.

Similarly, denote the $i$ th mode norm of a healthy structure by $G_{m h i}$, and the $i$ th mode norm of a damaged structure by $G_{m d i} 2$. The $i$ th mode index of the structural damage is defined as a weighted difference between the $i$ th mode norm of 
a healthy and damaged structure, i.e.,

$$
m i=G_{m h i} \stackrel{2}{2}-G_{m d i} \stackrel{2}{2} / G_{m h i} \stackrel{2}{2} .
$$

The $i$ th mode index reflects impact of the structural damage on the $i$ th mode.

The question arises how to measure the sensor and modal norms. It follows from equation (6) that the norm is determined from the system natural frequencies, modal damping ratios, and the modal input and output gains. The gains, on the other hand, are determined from the natural mode shapes at the actuator and sensor locations. Thus the measurements of natural frequencies, modal damping, and the modal displacements at the actuator and sensor locations of healthy and damaged structures allows for the localization of a structural damage. Note that the natural mode shapes characterize the structure itself since they are determined from the modal matrix $\Phi$. Thus, they depend neither on the input time history, $\mathrm{u}(\mathrm{t})$, nor on the actual system deformations, $\mathrm{q}(\mathrm{t})$.

\section{EXAMPLE}

A beam structure with fixed corners as in Figure 1 is analyzed. The cross-section area of the steel beams is of $1 \mathrm{~cm}^{2}$. Two damage cases are considered. First, as a $20 \%$ reduction of the stiffness of the beam No. 5 , and the second case as a $20 \%$ reduction of the stiffness of the beam No. 17. The structure was more densely divided near the damage locations to better reflect the stress concentration. Nineteen strain-gage sensors are placed at the beams 1-19. A vertical force applied at node $P$ excites the structure.

For the first case the sensor and the modal indices are shown in Figures 2(a,b). The sensor indices in Figure 2(a) indicate that the sensor No. 5, located at the damaged beam suffered the most changes. The modal indices in Figure 2(b) show that the first mode was heavily affected by the damage.

The sensor and modal indices for the second case are shown in Figure 3(a,b). Figure 3(a) shows the largest sensor index at location No. 17 of the damaged beam. The modal indices in Figure 3(b) show that the tenth and the second modes were mostly affected by the damage.

\section{REFERENCES}

1. M. I. Friswell, J. E. T. Penny and S. D. Garvey 1998 Computers and Structures 69, 547-556. A combined genetic and sensitivity algorithm for the location of damage in structures.

2. C.-P. Fritzen, D. Jennewein and T. Kiefer 1998 Mechanical Systems and Signal Processing 12, 163-186. Damage detection based on model updating methods.

3. T. Marwala and P. S. Heyns 1998 AIAA Journal, 36, 1494-1501. Multiple-criterion method for determining structural damage.

4. A. Messina, E. J. Williams and T. Contursi 1998 Journal of Sound and Vibration 216, 791-808. Structural damage detection by a sensitivity and statistical-based method.

5. Z. Y. SHI and S. S. LAW 1998 Journal of Sound and Vibration 218, 825-844. Structural damage localization from modal strain energy change.

6. S. K. Thyagarajan, M. J. Schultz and P. F. Pai 1998 Journal of Sound and Vibration 210, 162-170. Detecting structural damage using frequency response function.

7. W. Gawronski 1998 Dynamics and Control of Structures: A Modal Approach, New York Springer. 